\title{
Transmission Near-Field Scanning Optical Microscopy Investigation on Cellular Uptake Behavior of Iron Oxide Nanoparticles
}

\author{
Yu Zhang • Jennifer Reiber Kyle • Miro Penchev • \\ Vahid Yazdanpanah • Jinjiang Yu • Yi Li • Mo Yang • \\ Gurer Budak • Ekmel Ozbay • Mihrimah Ozkan • \\ Cengiz S. Ozkan
}

Published online: 7 June 2012

(C) Springer Science+Business Media, LLC 2012

\begin{abstract}
Cellular uptake behavior of iron oxide nanoparticles is investigated using a transmission near-field scanning optical microscopy (NSOM) without the need of fluorescent labeling. By using the transmission NSOM
\end{abstract}

Y. Zhang • V. Yazdanpanah • C. S. Ozkan $(\bowtie)$

Department of Mechanical Engineering,

University of California Riverside,

Riverside, CA 92521, USA

e-mail: cozkan@engr.ucr.edu

\section{Y. Zhang $\cdot$ Y. Li}

Institute of Textiles and Clothing,

the Hong Kong Polytechnic University,

Hong Kong, China

J. R. Kyle $\cdot$ M. Penchev $\cdot$ M. Ozkan

Department of Electrical Engineering,

University of California Riverside,

Riverside, CA 92521, USA

J. Yu $\cdot$ M. Yang

Department of Health Technology and Informatics,

Hong Kong Polytechnic University,

Hong Kong, China

G. Budak

Nanomedicine Research Center, Gazi University,

Besevler,

Ankara, Turkey

\section{E. Ozbay}

Nanotechnology Research Center, Department of Physics, Bilkent University,

Ankara, Turkey system, we could simultaneously explore the near-field optical analysis of the cell interior and record the topographic information of the cell surface. The cell endocytosis of iron oxide nanoparticles by normal breast MCF10A cells is first studied by this transmission NSOM system, and this dual functional nanoscale-resolution microscopy shows the capability of mapping the spatial localization of nanoparticles in/outside cell surface without the need of fluorescence labeling. Nanoscale optical signature patterns for iron oxide nanoparticle-loaded vesicles inside the cells were observed and analyzed.

Keywords AFM · NSOM - Iron oxide · Nanoparticle . Endocytosis $\cdot$ MCF10A

\section{Introduction}

The substantial investigation of nanoparticles recognition and uptake by biological cells is of utmost importance for their current technological and biomedical applications. In order to understand the interaction between nanoparticles and biological cells, it is crucial to be able to detect and localize them. The most popular methods to visualize nanoparticle within cells are fluorescence microscopy and transmission electron microscopy (TEM). TEM is one popular imaging method for studying the interactions between nanoparticles and cells at a superior nanoscale resolution. However, the time-consuming sample preparation process to get cell sections for TEM analysis hampered its wide usage, and 
only small regions of cells could be analyzed. Fluorescence microscopy is the primary imaging method for cell biologists. But for nanoparticle intracellular studies, the maximum resolution is limited by half of the wavelength based on fundamental diffraction limit [1]. Novel fluorescence microscopy methods such as photoactivated localization microscopy (PALM), stochastic optical reconstruction microscopy (STORM), and stimulated emission depletion (STED) have been developed to overcome the diffraction limit [2]. By utilizing a subwavelength dimension aperture on the scanning probe, near-field scanning optical microscopy (NSOM) also provides the capability to view biological samples in nanoscale through evanescent waves [3, 4]. Cellular imaging using fluorescent near-field optical microscopy has gained much interest due to the high sensitivity, noninvasiveness, and high spatial resolution [4]. Fluorescent NSOM is especially suited for selective excitation of fluorescence from cell plasma membrane [5], and many researchers used fluorescent NSOM to study nanometric-sized cell plasma membrane domains including singe molecules, proteins, lipids, and ion channel clusters [6-8]. However, to be visualized by fluorescencebased microscopies, nanoparticles generally need to be fluorescent, and it is a significant problem for nanoparticles without intrinsic optical fluorescence properties, such as iron oxide nanoparticles, titanium dioxide nanoparticles, silica nanoparticles, which have a wide range of applications [9]. To label nanoparticles or incorporate nanoparticles with fluoresence, the physicochemical surface properties of nanoparticles will likely be changed which will have a big impact over nanoparticle-cell interaction [9-12].

In this study, without the need of fluorescence labeling, we characterize the interaction between iron oxide nanoparticles and MCF10A normal breast epithelial cells based on transmission mode NSOM. This method allows us to simultaneously record high-resolution transmitted optical information and topographic information. The inhomogeneities appearing in transmission near-field optical image of cells may indicate the spatial localization of iron oxide nanoparticles due to their significant different absorption properties from cells. Iron oxide nanoparticles in this study have been widely used in the following biomedical applications such as magnetic resonance imaging, targeting drug delivery, hyperthermia inducing agent, tissue repair, detoxification, and cell separation [13-16].

\section{Materials and Methods}

\subsection{Cell Culture}

Human breast normal epithelial cells (MCF10A) were purchased from American Type Culture Collection (ATCC) and maintained in mammary epithelial cell medium (MEGM,
BulletKit, Cambrex) supplemented with $100 \mathrm{ng} / \mathrm{ml}$ cholera toxin (EMD, Biosciences) at $37^{\circ} \mathrm{C}$ in a humidified and $5 \%$ $\mathrm{CO}_{2}$ incubator. For NSOM microscopy, cells were grown on glass substrates with a thickness of $100 \mu \mathrm{m}$ in Petri dishes. Thin glass substrates were required for transmission mode imaging in NSOM.

\subsection{Nanoparticle Characterization}

The magnetic iron oxide nanoparticles $\left(\gamma-\mathrm{Fe}_{2} \mathrm{O}_{3}\right)$ were purchased from Alfa Aesar and the average diameter is around $30 \mathrm{~nm}$. The nanoparticles were imaged by scanning electron microscopy (SEM, JEOL, JSM-6300). Ultraviolet-visible (UV-VIS) absorbance measurements of iron oxide nanoparticles were performed on a PerkinElmer Lambda $35 \mathrm{UV}-$ Vis spectrometer. Zeta potential measurements of iron oxide nanoparticles suspended in $1 \times$ phosphate buffered saline (PBS) buffer were performed on a zeta potential analyzer (ZetaPALS) from Brookhaven Instruments.

\subsection{Sample Preparation}

Magnetic iron oxide nanoparticles $(50 \mu \mathrm{g} / \mathrm{ml})$ were incubated with MCF10A cells at $37^{\circ} \mathrm{C}$ in a humidified and $5 \% \mathrm{CO}_{2}$ for $30 \mathrm{~min}, 4 \mathrm{~h}$, and $24 \mathrm{~h}$. Cells incubated with iron oxide nanoparticles were then washed extensively with $1 \times$ PBS for three times. Cells were then fixed by $1 \%$ formaldehyde in $1 \times$ PBS at room temperature for $10 \mathrm{~min}$, washed with $1 \times$ PBS for three times, followed by washing with an increasing graded ethanol series to dehydrate and then left in vacuum dessicator.

\subsection{Transmission NSOM Imaging}

In this work, all of the samples were examined using the Nanonics Multiview 1000 system by Nanonics Imaging under ambient conditions. This system is an integrated microscopy system including conventional farfield imaging, atomic force microscope and near-field scanning optical microscope. Cantilevered probe with an aperture diameter of $50 \mathrm{~nm}$ with optical fibers (Nanonics, Israel) were used for nanoparticle and cell imaging. The transmission and reflection mode in NSOM are based on how the light is collected by the detector. For this article, we utilized transmission mode NSOM to collect light after it passes the sample using a $488 \mathrm{~nm}$ Argon-ion laser source (Laser Physics, UT, USA). The transmitted light was collected with a $50 \times$, NA: 0.45 objective (Olympus SLMPLAN, Japan) and detected with a photon counting module (Perkin-Elmer, USA). All the images were processed with WSxM image processing software. 


\subsection{Transmission Electron Microscopy}

MCF10A cells were first cultured on Thermanox plastic cover slips. Then cells were incubated with $50 \mu \mathrm{g} / \mathrm{ml}$ iron oxide nanoparticles dispersions for $30 \mathrm{~min}, 4 \mathrm{~h}$, and $24 \mathrm{~h}$. Cells were first rinsed with $1 \times$ PBS for three times after incubation, rinsed with $0.1 \mathrm{M}$ Sodium cacodylate, fixed in $2 \%$ Gluteraldahyde in the same buffer, and then post fixed with $1 \%$ osmium tetroxode. After dehydrated using an increasing graded ethanol series, cells were embedded in Spuur resin to polymerize. The specimens were then sectioned using a Sorvall MT2 Ultramicrotome and poststained with uranyl acetate and lead citrate. Finally, TEM micrographs were obtained by a TEM Tecnai12 at Central Facility for Advanced Microscopy and Microanalysis (CFAMM) in the University of California, Riverside.

\subsection{Analysis of NSOM Images}

Specific optical features in the NSOM images were analyzed by integrated morphology analysis module of MetaMorph ${ }^{\circledR}$ imaging system (Nikon). Small clusters of nanoparticles were selected by a contrast threshold on the boundary of the selected active regions. Each active region profile gave both area and contrast intensity information. The contrast intensities of the selected cluster regions are measured relative the surrounding background. Here, the Michelson contrast was used for the analysis of nanoparticle clusters:

$C_{\mathrm{M}}=\frac{L_{\mathrm{Max}}-L_{\mathrm{Min}}}{L_{\mathrm{Max}}+L_{\mathrm{Min}}}$

where $C_{\mathrm{M}}$ is the Michelson contrast, $L_{\mathrm{Max}}$ is the maximum luminance within the domain, and $L_{\mathrm{Min}}$ is the minimum luminance on the boundary. All the area and contrast intensity data were automatically collected for statistical analysis and histogram binning.

\section{Results and Discussion}

Tapping mode was utilized to scan cell samples in this study, which is particularly sensitive to changes in height and good for soft cell samples because the effect of lateral forces introduced in the contact mode is eliminated [17, 18]. To investigate nanoparticle endocytosis by biological cells, transmission mode NSOM is preferred. The hypothesis is that due to the difference in absorption for different materials, contrast difference in transmission near-field optical image will give more information than reflection near-field optical image. Because of the obvious absorption difference between iron oxide nanoparticles and cells, an obvious contrast difference is supposed to appear in transmission near-field optical image. The iron oxide nanoparticles expect to absorb more laser light than cells. Thus, nanoparticle endocytosis might be directly visualized in the near-field optical image without the need of fluorescent labeling. Furthermore, the simultaneously obtained topographical images will give complementary information. The setup and schematic illustration of transmission NSOM to investigate the nanoparticle endocytosis by biological cells is described in Fig. 1. In transmission mode NSOM, by locating the probe tip near the sample, which induces scattering of evanescent near-field sample, evanescent wave is converted into a propagating wave carrying light signal near sample with a nanoscale resolution. By collecting the scattered field after it passes the sample, absorbance-based transmission mode NSOM image is formed.

The topographic image and transmission near-field optical image of iron oxide nanoparticles are shown in Fig. 2a and $b$. The cluster of iron oxide nanopartices shows some dark regions and bright spots in transmission NSOM image. The dark regions indicate the lower transmission intensity of collected laser light due to iron oxide nanoparticle adsorption. At the same time, some bright spots are also observed in transmission NSOM image, which is likely caused by the laser light scattering from the iron oxide nanoparticle surfaces. Fig. 2c shows the UV-VIS absorption full-range spectrum of iron oxide nanoparticles from 200 to $1000 \mathrm{~nm}$, and the adsorption at $488 \mathrm{~nm}$ is still in a high level although no adsorption peak is observed. Besides, the SEM image of iron oxide nanoparticles is shown in Fig. 2d. We also performed zeta potential measurements of iron oxide nanoparticles in $1 \times$ PBS buffer. The zeta potential value of these iron oxide nanoparticles is $-30.41 \pm 2.83 \mathrm{mV}$, which indicated a good dispersion of these anionic iron oxide nanoparticles.

The simultaneously collected topographic image and transmission near-field optical image of MCF10A cells without incubation with iron oxide nanoparticles are shown in Fig. 3a and b. Fig. 3c and d, Fig. 3e and f, and Fig. 4a and $\mathrm{b}$ are the topographic images and transmission near-field optical images for MCF10A cells after incubated with $50 \mu \mathrm{g} / \mathrm{ml}$ iron oxide nanoparticles suspension for $30 \mathrm{~min}$, $4 \mathrm{~h}$, and $24 \mathrm{~h}$, respectively. Fig. $4 \mathrm{c}$ and d shows the zoomins of topographic and near-field optical images boxed in Fig. $4 \mathrm{a}$ and b, respectively. Fig. $4 \mathrm{e}$ is a $3 \mathrm{D}$ topographic image of Fig. 4c after superimposed with near-field transmission optical image Fig. 4d. Although the topographic images of MCF $10 \mathrm{~A}$ cells are similar for cells without nanoparticle incubation and cells after iron oxide nanoparticles incubation for $30 \mathrm{~min}, 4 \mathrm{~h}$, and $24 \mathrm{~h}$, obvious differences are observed in their near-field optical images of cells. From near-field optical images Figs. 3f and $4 b$, dark spots with bright surrounding edges are observed and a larger amount of these patterns is observed in Fig. 4b. This kind 
Fig. 1 Block diagram of the transmission mode near-field optical microscope (NSOM). The NSOM probe is a cantilevered optical fiber and scans using a normal force feedback. Inset: A schematic illustration of transmission NSOM imaging on a cell loaded with iron oxide nanoparticles

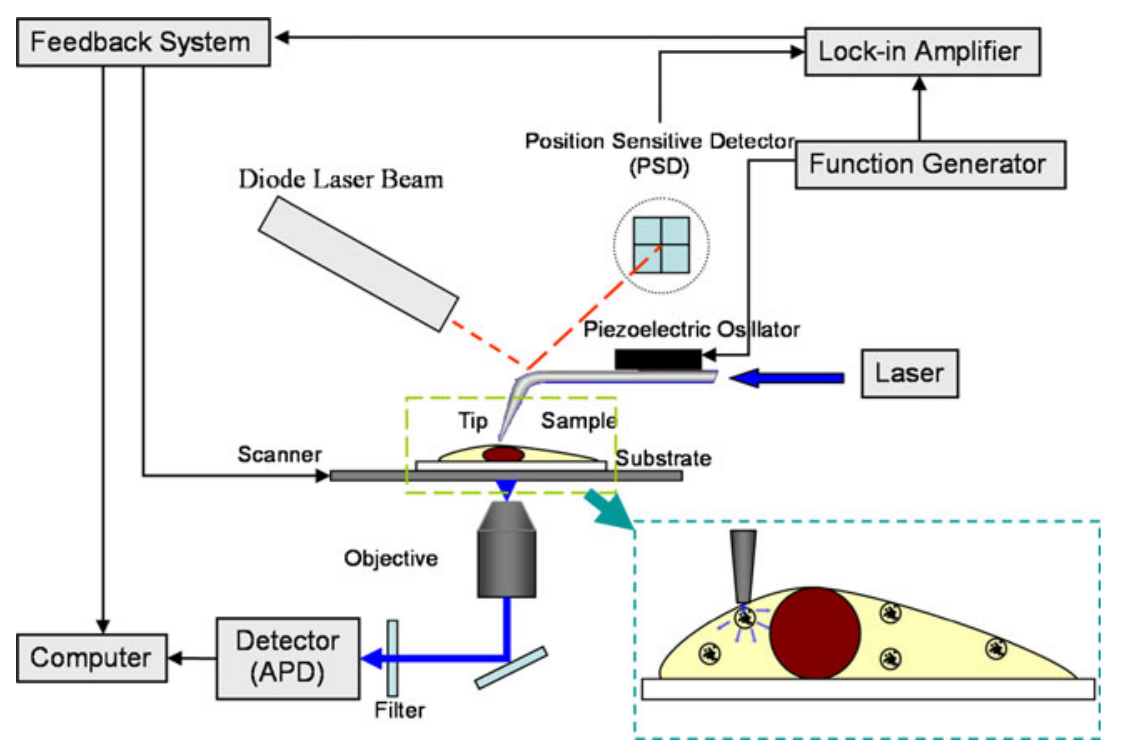

of pattern might indicate the iron oxide nanoparticles that were uptaken by cells through a vesicle-mediated transport, which is not observed in Fig. $3 b$ and d.

The receptor-mediated cell endocytosis of metal and oxide nanoparticles was confirmed to be induced by a nonspecific adsorption of proteins on nanoparticle surface from cell culture medium [19, 20]. During endocytosis, a small portion of the cell surface plasma membrane is invaginated to form a new intracellular vesicle to transport external substances inside the cells [21]. The cell cytoplasm, the fluids surrounding the iron oxide nanoparticles inside the vesicles and iron oxide nanoparticles have different refractive and absorbent indexes. This might be the reason why the iron oxide nanoparticles loaded in vesicles inside the
Fig. 2 Simultaneously collected topographic (a) and optical image (b) of iron oxide nanoparticles only by transmission NSOM with a $50 \mathrm{~nm}$ aperture tip utilizing a $488 \mathrm{~nm}$ argon laser. UV-VIS adsorption spectrum (c) and SEM image (d) of iron oxide nanoparticles

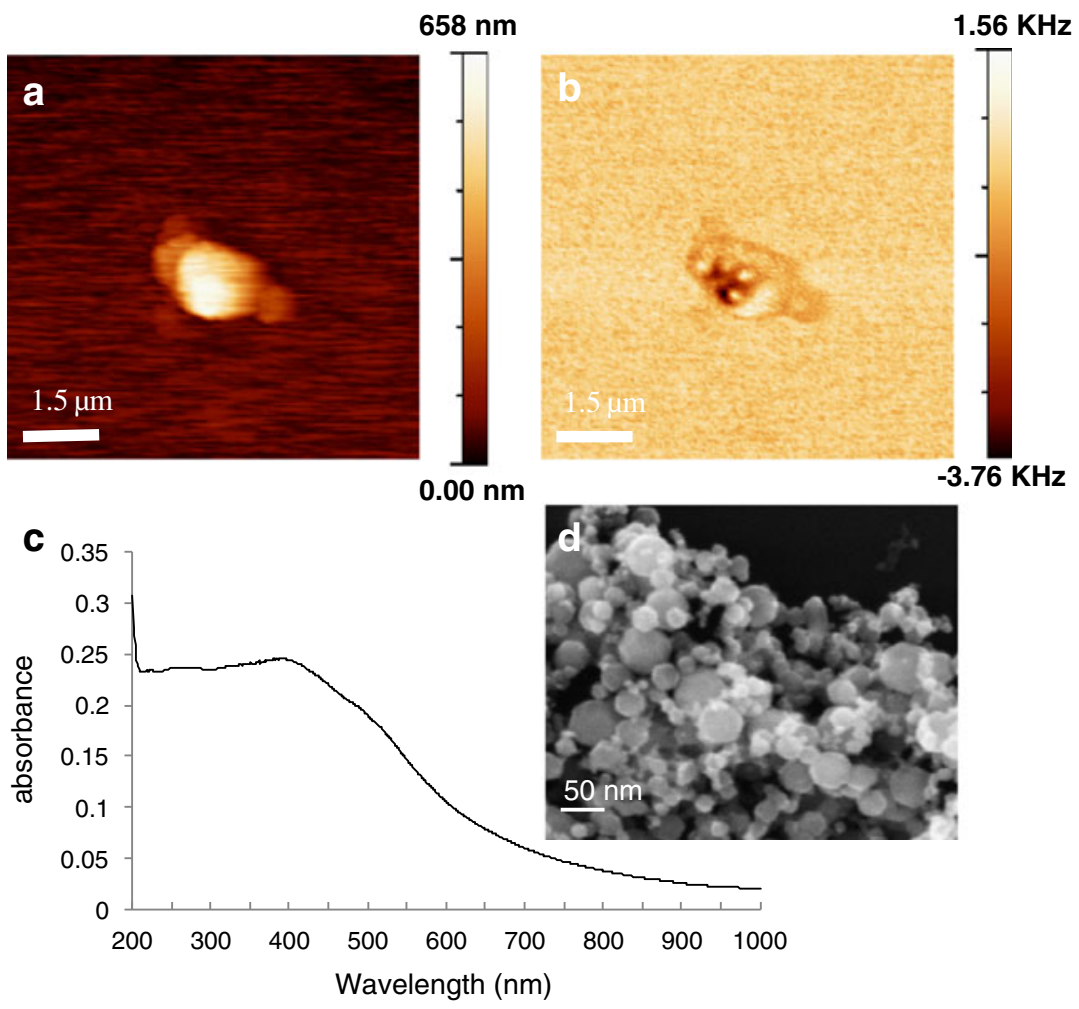


Fig. 3 Simultaneously collected topographic and optical images of MCF10A cells by transmission NSOM with a $50 \mathrm{~nm}$ aperture tip utilizing a $488 \mathrm{~nm}$ argon laser. Panels (a) and (b), (c) and (d), and (e) and (f) are topographic images and optical images for control cells, after incubated with iron oxide nanoparticles for $30 \mathrm{~min}$ and $4 \mathrm{~h}$, respectively. Colored circles indicate the locations of some nanoparticleloaded vesicles in both topographic and optical image of MCF10A cells after incubated with iron oxide nanoparticles for $4 \mathrm{~h}$
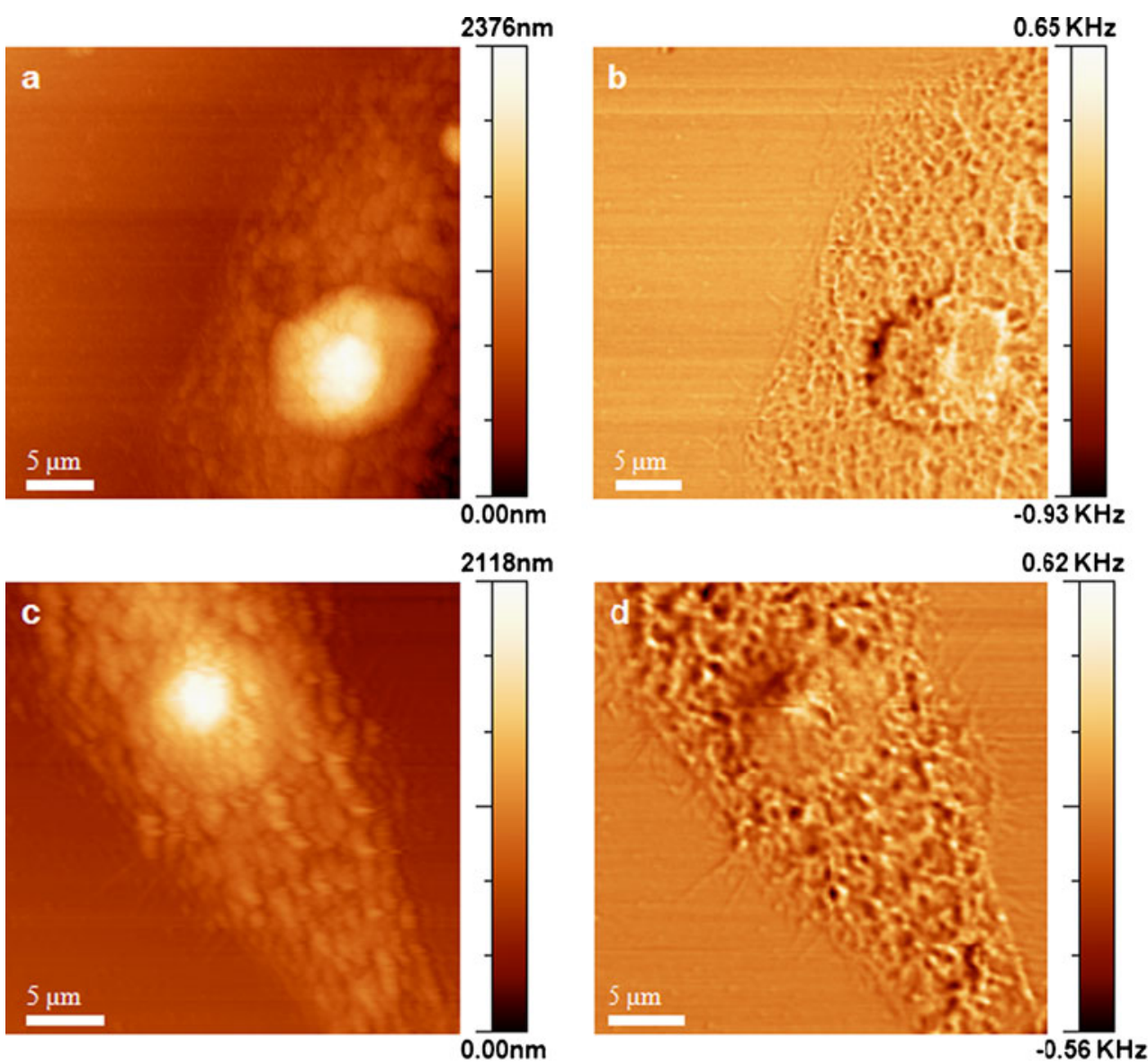

$955 \mathrm{~nm}$

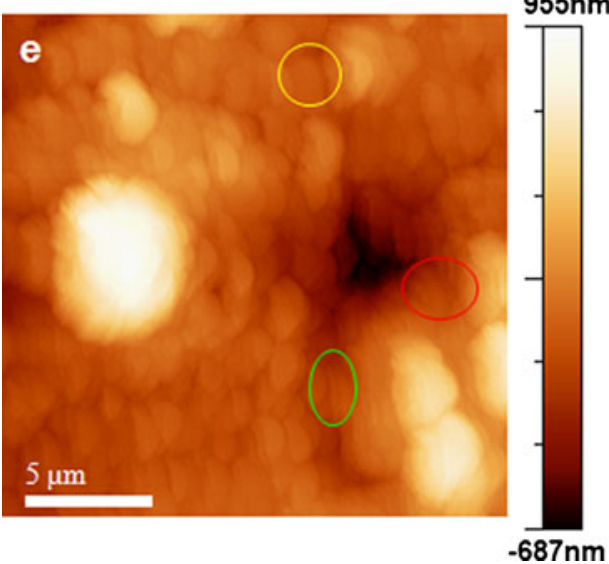

cells will appear as a dark spot with a bright surrounding edge. The bright surrounding edge might also be caused by the laser light scattering from iron oxide nanoparticles surfaces, in which we also observed some bright spots in transmission NSOM image of iron oxide nanoparticles cluster. From the near-field optical images, we observed this pattern in MCF10A cells after incubated with iron oxide nanoparticles for 4 and $24 \mathrm{~h}$ and did not observe it in cells without nanoparticle incubation and cells after nanoparticle incubation for $30 \mathrm{~min}$. We believe that this optical pattern (dark spot with a bright surrounding edge) is a signature pattern for nanoparticle-loaded vesicles inside the cells. For the nanoparticles binding on the cell membrane, the nearfield transmission images might observe bright spots for small-size nanoparticle clusters due to the scattered laser light or dark spots for big-sized nanoparticle clusters due to the absorbed laser light but without a bright surrounding edge.

TEM micrographs of MCF10A cells after incubated with iron oxide nanoparticles for $30 \mathrm{~min}, 4 \mathrm{~h}$, and $24 \mathrm{~h}$ are shown 
Fig. 4 Simultaneously collected topographic (a) and optical images (b) of MCF10A cells by transmission NSOM after incubated with iron oxide nanoparticles for $24 \mathrm{~h}$, scanned by a $50 \mathrm{~nm}$ aperture tip utilizing a $488 \mathrm{~nm}$ argon laser. $\mathbf{c}$ and $\mathbf{d}$ were obtained by zooming in the indicated area of $\mathbf{a}$ and $\mathbf{b}$. $\mathbf{e}$ $3 \mathrm{D}$ image of the zoom in area displays a combination of the topographic data and near-field optical data. Red arrows point out the boxed area $A$ and $B$.
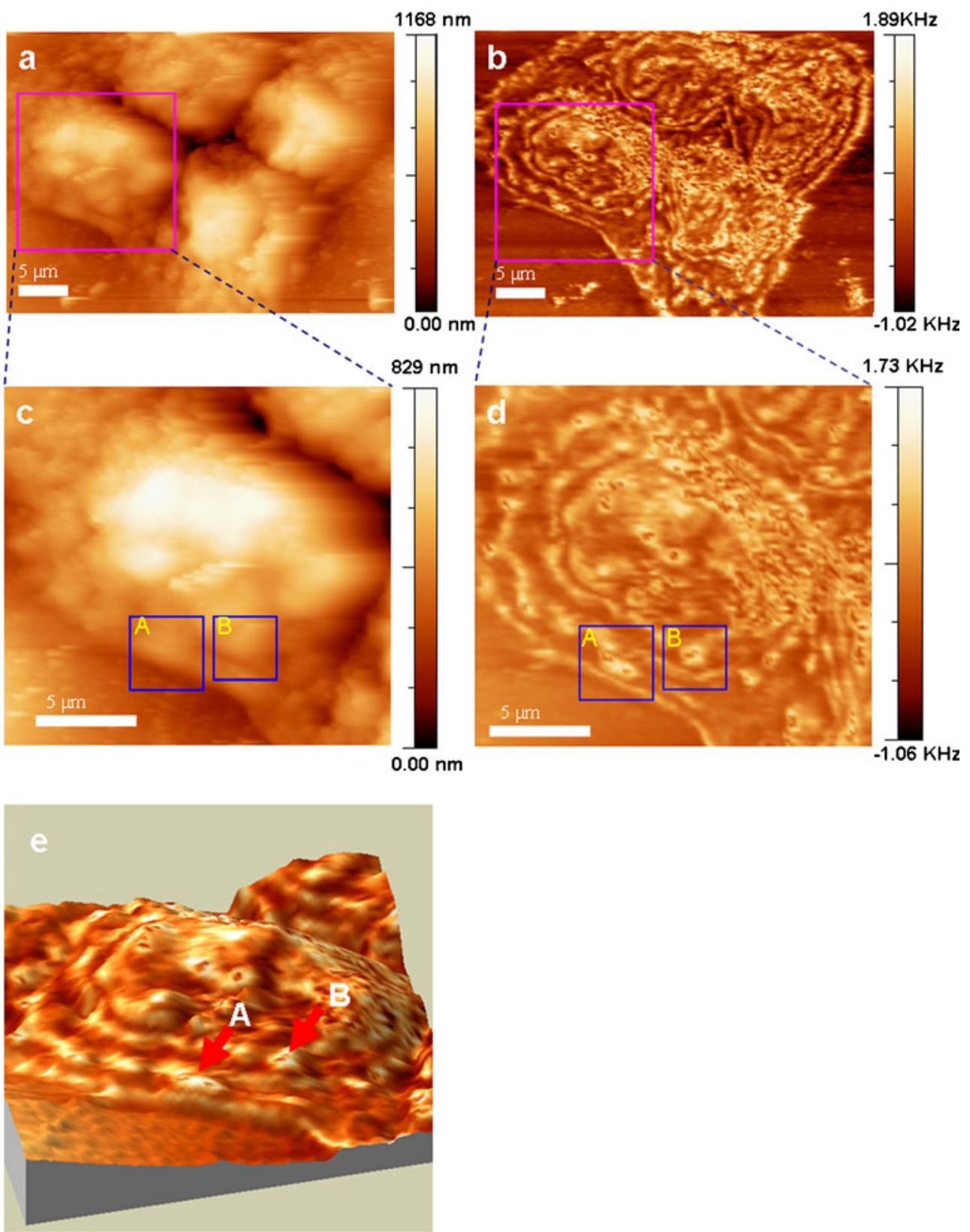

in Fig. 5a, b, c, and d, respectively. From TEM images, after 30 min incubation, iron oxide nanopartices are still binding on the cell membrane. After $4 \mathrm{~h}, \mathrm{MCF} 10 \mathrm{~A}$ cells have internalized iron oxide nanoparticles through a vesicular transport. Most of the vesicles in MCF10A cells observed in TEM images are also in the same size range of what is observed in near-field optical images. From TEM images, we observed that iron oxide nanoparticles aggregated before internalized by MCF 10A cells. The study by Wilhelm et al. $[20,22]$ suggested the clusters of anionic nanoparticles binding on the cell surfaces might be caused by the repulsive forces from the large negatively charged domains of the cell surface, and most aggregated nanoparticles will be internalized by cells through membrane invaginations. The nanoparticle clustering mechanism during cellular uptake has also been explained by Jin et al. [23], and they have developed a deterministic kinetic model for endocytosis and this model explains that nanoparticles aggregate on the cell membrane to form a size sufficient to generate a large enough enthalpic contribution via receptor-ligand interactions to overcome the elastic energy and entropic barriers associated with vesicle formation.

We utilized the topographic images and transmission near-field optical images for MCF10A cell after incubated with iron oxide nanoparticles for $24 \mathrm{~h}$ for a 
Fig. 5 TEM micrographs of MCF10A cells that were incubated with iron oxide nanoparticles for $30 \mathrm{~min}$ (a), $4 \mathrm{~h}$ (b), and $24 \mathrm{~h} \mathrm{(c)} \mathrm{and} \mathrm{(d)} \mathrm{at} 37^{\circ}$ C. All slices were treated with uranyl acetate to stain membranes and lead citrate to stain the nuclear body. Colored arrows represent selected cell organelles: nuclei (yellow), neucleolus (green), mitochondria (purple), and vesicles with iron oxide nanoparticles inside (red)
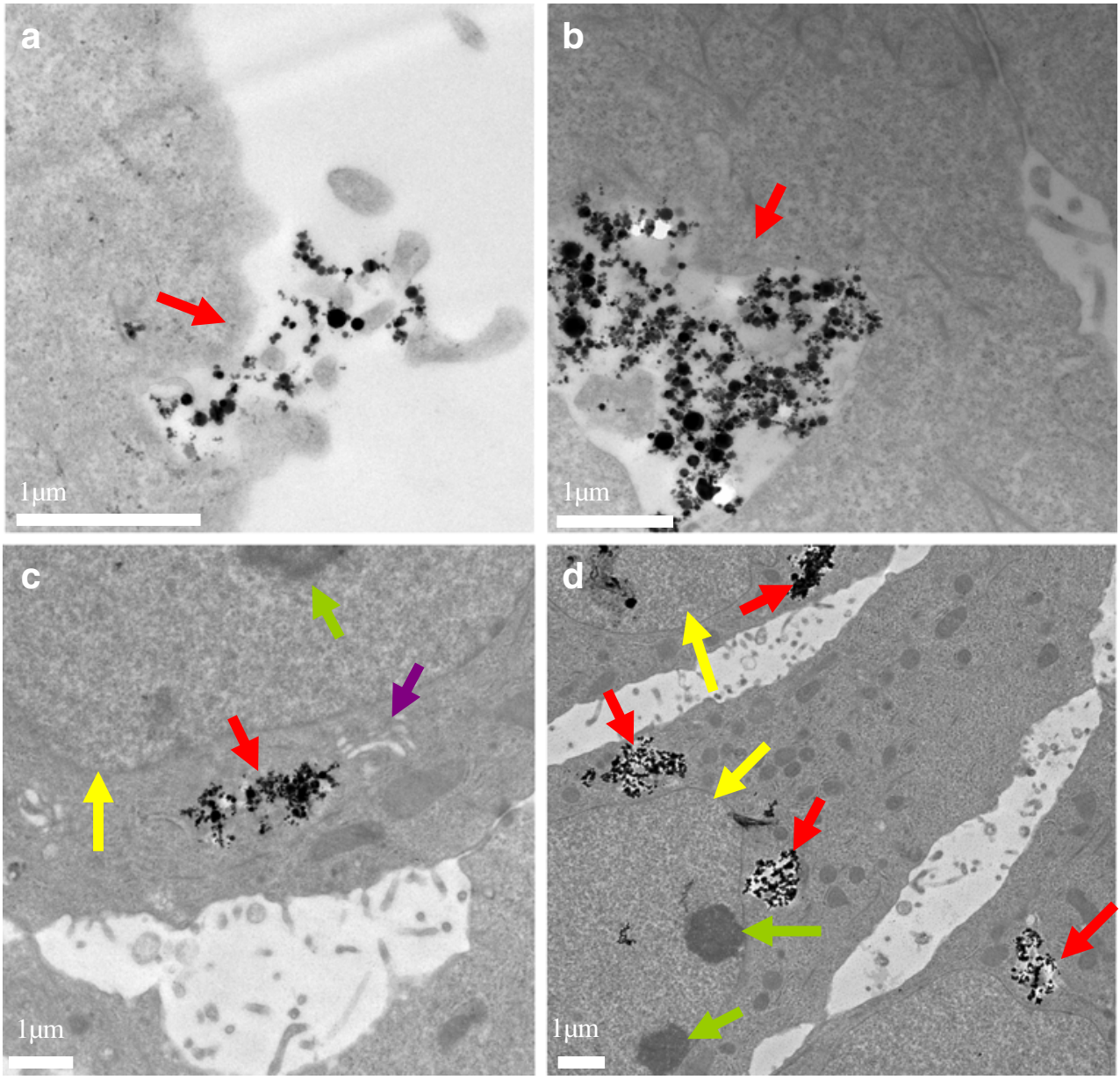

detailed line profile and statistical analysis. In Fig. 6a, the top diagram is a topography cross-section corresponding to the red line in area $A$ boxed in Fig. $4 \mathrm{c}$ and the bottom diagram is a transmitted light intensity profile corresponding to the same line. The information from these two line profiles indicates the existence of one iron oxide nanoparticle-loaded vesicle under the cell membrane. Similarly, in Fig. 6b, the top diagram is a topography cross-section corresponding to the yellow line in area $B$, and the bottom diagram is a transmitted intensity profile corresponding to the yellow line at the same position, which indicates another iron oxide nanoparticle-loaded vesicle. The arrows in transmitted intensity profile point out the nanoparticle clusters inside the vesicles.

For statistical analysis purpose, we used edge-finding routines of Adobe Photoshop (Adobe Systems, San Jose, CA) to define the edges of the nanoparticle clusters (Fig. 7a) and then overlay the domain boundaries to the near-field optical image (Fig. 7b). Using the rules described in "Materials and Methods," the edges of nanoparticle cluster domains were determined in
Fig. 6a. In Fig. 7c and d, the normalized histograms provide an expanded analysis of the equivalent diameters and the contrast intensities of these nanoparticle clusters. In this analysis, we set the threshold contrast as 0.1 . When the Michelson contrast is less than 0.1, the domain is not considered to be a cluster of nanoparticles. It was noted that most clusters of nanoparticles identified were around $300 \mathrm{~nm}$. Larger clusters were also observed up to $1.5 \mu \mathrm{m}$.

The size of the nanoparticle clusters we got from near-field optical images may not be accurate due to the limited NSOM optical detection depth. The optical detection sensitivity of NSOM is mainly determined by the aperture dimensions of tips and the depth of nearfield penetration into the specimen [3]. Only those nanoparticle-loaded vesicles, which are close to the cell surface, could be observed. Despite the detection limit, we could see that AFM/NSOM hybrid microscopy offers a powerful label-free method to study the interaction between cells and nanoparticles yet with a much higher resolution compared with the conventional optical microscope. 
Fig. 6 a The top diagram is a topography cross-section corresponding to the indicated line in area $A$, and the bottom diagram is a transmitted intensity profile corresponding to the indicated line at the same position. b The top diagram is a topography cross-section corresponding to the indicated line in area $B$, and the bottom diagram is a transmitted intensity profile corresponding to the indicated line at the same position. Inset: Images of AFM or NSOM images of boxed area $A$ and $B$ a

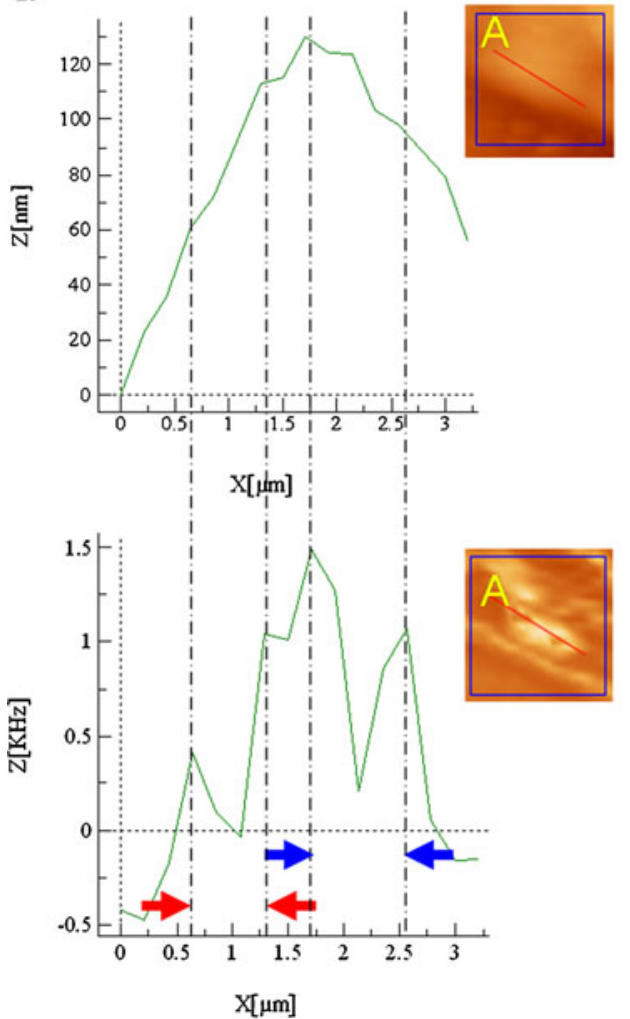

b

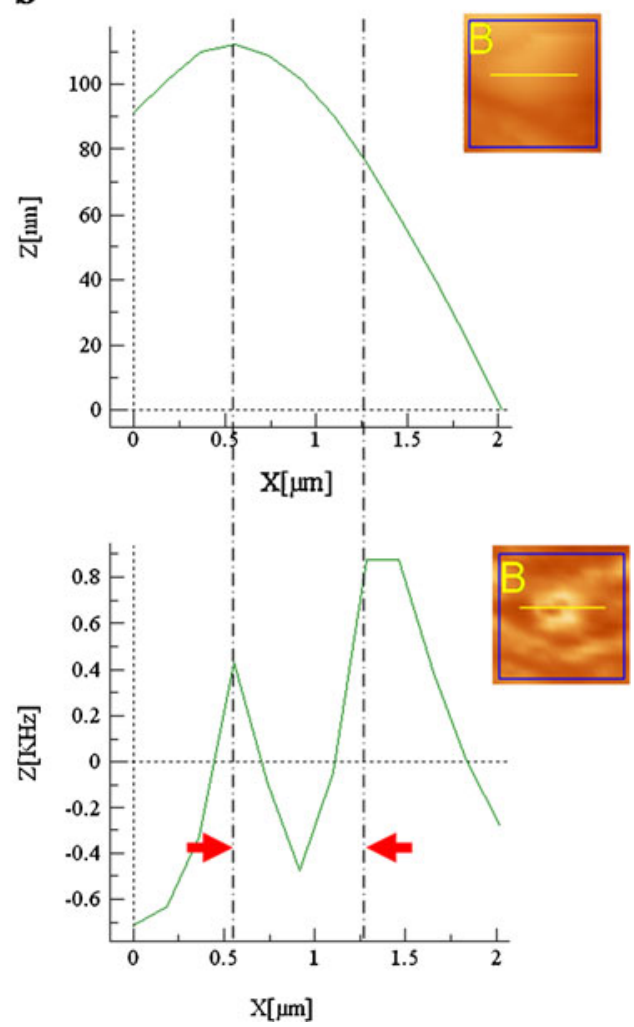

\section{Conclusion}

We examined the MCF10A normal epithelial cells and their interactions with iron oxide nanoparticles using a transmission NSOM system. The interaction between nanoparticles and cells could be easily studied and mapped without the need of fluorescent labeling on the nanoparticles. Optical signature patterns (dark spots with bright surrounding edges) for nanoparticle-loaded vesicles inside the cells were observed. Overall, this imaging method provides a powerful
Fig. 7 a The edges of the nanoparticle clusters are defined using edge-finding routines of Adobe Photoshop (Adobe Systems) and $\mathbf{b}$ an overlay image of the domain boundaries and NSOM image. Histogram displays of distributions of small clusters of nanoparticles c average diameters of domains and d contrast measured by the edges intensity differences
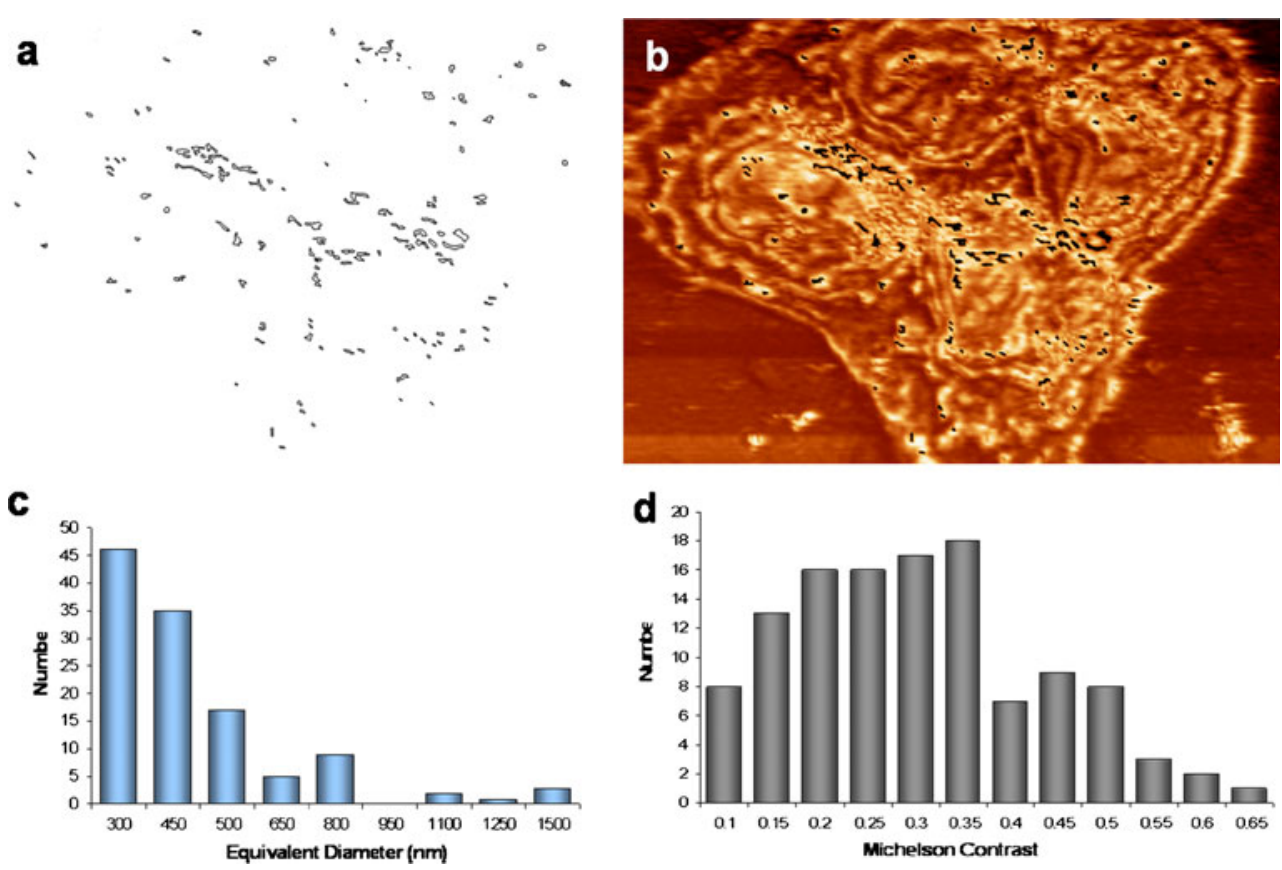

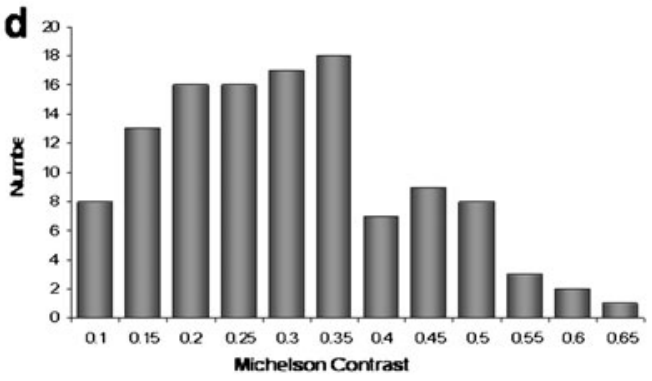


fluorescence label-free method to study the interaction between cells and nanoparticles with a much higher resolution compared with the conventional optical microscope.

Acknowledgment We gratefully acknowledge the Center of Excellence of Nanotechnology for Treatment, Understanding, and Monitoring of Cancer (NANOTUMOR) for providing financial support for this project. Mr. Stephen McDaniel in the Central Facility for Advanced Microscopy and Microanalysis (CFAMM) at the University of California is gratefully acknowledged for assistance in preparing the microtome cell slides for TEM.

\section{References}

1. Pawley, J. B. (Ed.). (1995). Handbook of biological confocal microscopy. New York: Plenum.

2. Zenobi, R. (2008). Analytical and Bioanalytical Chemistry, 390, 215.

3. Betzig, E., \& Trautman, J. K. (1992). Science, 257, 189.

4. de Lange, F., Cambi, A., Huijbens, R., de Bakker, B., Rensen, W., Garcia-Parajo, M., van Hulst, N., Figdor, C. G. (2001). Journal of Cell Science, 114, 4153.

5. Enderle, T., Ha, T., Chemla, D. S., Weiss, S. (1998). Ultramicroscopy, 71, 303.

6. Hwang, J., Gheber, L. A., Margolis, L., Edidin, M. (1998). Biophysical Journal, 74, 2184.

7. Ianoul, A., Street, M., Grant, D., Pezacki, J., Taylor, R. S., Johnston, L. J. (2004). Biophysical Journal, 87, 3525.

8. Koopman, M., Cambi, A., de Bakker, B. I., Joosten, B., Figdor, C. G., van Hulst, N. F., Garcia-Parajo, M. F. (2004). FEBS Letters, 573,6 .
9. Thurn, K. T., Paunesku, T., Wu, A., Brown, E. M., Lai, B., Vogt, S., Maser, J., Aslam, M., Dravid, V., Bergan, R., Woloschak, G. E. (2009). Small, 5, 1318.

10. Maxwell, D. J., Bonde, J., Hess, D. A., Hohm, S. A., Lahey, R., Zhou, P., Creer, M. H., Piwnica Worms, D., Nolta, J. A. (2008). Stem Cells, 26, 517.

11. H. Mader, X. H. Li, S. Saleh, M. Link, P. Kele, O. S.Wolfbeis. Fluorescence methods and applications: Spectroscopy, imaging, and probes. Wiley-Blackwell. 218 (2008).

12. Lee, J. H., Schneider, B., Jordan, E. K., Liu, W., Frank, J. A. (2008). Advanced Materials, 20, 2512.

13. Clark, P. R., Chua-Anusorn, W., St Pierre, T. G. (2003). Magnetic Resonance in Medicine, 49, 572.

14. Gupta, A. K., \& Gupta, M. (2005). Biomaterials, 26, 3995.

15. Hergt, R., Andra, W., d'Ambly, C. G., Hilger, I., Kaiser, W. A., Richter, U., Schmidt, H. G. (1998). IEEE Transactions in Magnetics, 34, 3745.

16. Lee, H., Lee, E., Kim, D. K., Jang, N. K., Jeong, Y. Y., Jon, S. (2006). Journal of the American Chemical Society, 128, 7383.

17. Frankel, D. J., Pfeiffer, J. R., Surviladze, Z., Johnson, A. E., Oliver, J. M., Wilson, B. S., Burns, A. R. (2006). Biophysical Journal, 90, 2404.

18. Stark, M., Moller, C., Muller, D. J., Guckenberger, R. (2001). Biophysical Journal, 80, 3009.

19. Chithrani, B. D., Ghazani, A. A., Chan, W. C. W. (2006). Nano Letters, 6, 662.

20. Wilhelm, C., Gazeau, F., Roger, J., Pons, J. N., Bacri, J. C. (2002). Langmuir, 18, 8148 .

21. G.M. Cooper (Ed.). The cell: A molecular approach. ASM Press, Washington D.C. (2000)

22. Wilhelm, C., Billotey, C., Roger, J., Pons, J. N., Bacri, J. C., Gazeau, F. (2003). Biomaterials, 24, 1001.

23. Jin, H., Heller, D. A., Sharma, R., Strano, M. S. (2009). ACS Nano, $3,149$. 